\title{
Preliminary evidence of an association between vitamin $D$ status and self-assessed sleep duration but not overall sleep quality: results from the D-FINES study of South Asian and Caucasian pre- and post-menopausal women living in Southern England
}

\author{
A. L. Darling, D. J. Skene and S. A. Lanham-New \\ Faculty of Health and Medical Sciences, University of Surrey, Guildford GU2 7XH, UK
}

\begin{abstract}
Vitamin D status has been associated in recent years with an increasing variety of chronic diseases. Indeed, evidence is emerging that it may play a role in a wide range of diseases such as cancer, heart disease, autoimmune disease, dementia and diabetes ${ }^{(1)}$. Recent preliminary analysis of the 2005-2006 N-HANES (National Health and Nutrition Examination Survey) data in the US suggests vitamin D deficiency may be associated with shorter sleep duration ${ }^{(2)}$. However, to the authors' knowledge this result has not been re-tested in other datasets, or in other ethnic groups. Therefore, the aim of this study was to investigate whether there is an association between vitamin D status and self-reported sleep quality using data obtained from the D-FINES (vitamin D, Food Intake, Nutrition and Exposure to Sunlight in Southern England) study. In summer 2006, $n 375$ South Asian (A) and Caucasian (C) premenopausal (PRM) and post-menopausal (POM) women were assessed for vitamin D status (serum $25(\mathrm{OH}) \mathrm{D})$ as well as relevant anthropometric and lifestyle information. A subset of the original participants ( $n 33 \mathrm{~A}$ and $n$ 80C) were re-assessed again in summer 2010 and also self-completed the PSQI (Pittsburgh Sleep Quality Index), which is a validated method for assessing self-reported sleep quality ${ }^{(3)}$.

Partial correlations were run using PASW 18.0 to examine associations between 25(OH)D status (Summer 2006 results were used as Summer 2010 results were not yet available) and overall PSQI score, as well as between 25(OH)D and the subscales of the PSQI. The analysis controlled for the potential confounders of social deprivation index, age, physical activity and BMI. 25(OH)D and PSQI scores were skewed so were log transformed prior to analysis. Correlations were run with all participants pooled together, and also in separate ethnic and menopausal subgroups (see Table). No significant association $(P \geq 0.05)$ was found between 25(OH)D and total PSQI score for any of the participant subgroups. Also, no significant association was found between 25(OH)D and any of the PSQI subscales in any of the subgroups (data not shown), except for sleep duration, which in all participants together was statistically significant $(r=-0.261$, $P=0.01, \mathrm{df}=92)$ and of borderline significance for PRM women $(r=-0.402, P=0.07, \mathrm{df}=19)$. Also, a statistically significant result was found between 25(OH)D and habitual sleep efficiency in PRM women only $(r=-0.455, P=0.04, \mathrm{df}=19)$.
\end{abstract}

Partial correlations between vitamin D status and total PSQI score by participant subgroups

\begin{tabular}{|c|c|c|c|c|c|c|c|c|c|}
\hline & & $\begin{array}{l}\text { ALL } \\
\text { POM }\end{array}$ & $\begin{array}{l}\text { ALL } \\
\text { PRM }\end{array}$ & $\begin{array}{c}\mathrm{POM} \\
\mathrm{A}\end{array}$ & $\begin{array}{c}\text { PRM } \\
\text { A }\end{array}$ & $\begin{array}{c}\text { ALL } \\
\text { A }\end{array}$ & $\begin{array}{c}\text { POM } \\
\text { C }\end{array}$ & $\begin{array}{c}\text { PRM } \\
\text { C }\end{array}$ & $\begin{array}{c}\text { ALL } \\
\text { C }\end{array}$ \\
\hline$r$ & -0.157 & -0.068 & -0.362 & -0.034 & * & -0.148 & 0.065 & -0.075 & 0.025 \\
\hline$p$ & 0.14 & 0.59 & 0.13 & 0.92 & $*$ & 0.59 & 0.65 & 0.80 & 0.84 \\
\hline$N$ & 90 & 67 & 23 & 13 & 5 & 18 & 54 & 18 & 72 \\
\hline
\end{tabular}

Therefore, this preliminary analysis supports no association between vitamin D status and overall self-reported sleep quality in either of the ethnic groups studied. However, it does lend support to findings in the literature ${ }^{(2)}$ of an association between vitamin D status and sleep duration in particular. However, it must be borne in mind that this may due to the confounding effects of light exposure and musculoskeletal pain on any relationship between vitamin D and sleep. Indeed, repeat analysis using the most recent measure of $25(\mathrm{OH}) \mathrm{D}$ status, and adjusting for light exposure, general health and musculoskeletal pain as possible confounders is currently in progress.

ALD is recipient of a University of Surrey PhD Scholarship. The D-FINES Study was funded by the FSA (NO5064).

1. Holick MF (2011) J Investig.Med (Epublication ahead of print version).

2. Pande R, Chandrasekhar R, Kaplish N et al. (2009) Sleep 32S, A136.

3. Buysse DJ, Reynolds CF, Monk TH et al. (1989) Psychiatry Res 28, 193-213. 